\title{
Local Stability Analysis of a Mathematical Model of the Interaction of Two Populations of Differential Equations (Host-Parasitoid)
}

\author{
Dewi Anggreini \\ Lecturer of Study Program Mathematics Education, STKIP PGRI Tulungagung \\ Jl. Mayor Sujadi Timur No. 7 Tulungagung, 66221 Tel/Fax: (0355) 321426
}

Author correspondency:

anggreini_004@yahoo.com

\begin{abstract}
Mathematical model has many benefits in life, especially the development of science and application to other fields. The mathematical model seeks to represent real-life problems formulated mathematically to get the right solution. This research is the application of mathematical models in the field of biology that examines the interaction of the two populations that host populations and parasitoid populations. This study differs from previous studies that examine the interaction of two more species that prey and predators where predators kill prey quickly. In this study the parasitoid population slowly killing the host population by living aboard and take food from the host population it occupies. In this study of differential equations are used to construct a mathematical model was particularly focused on the stability of the local mathematical model of interaction of two differential equations that host and parasitoid populations. Stability discussed in this study are stable equilibrium points are obtained from the characteristic equation systems of differential equations host and parasitoid interactions. Type the stability of the equilibrium point is determined on the eigenvalues of the Jacobian matrix. Analysis of stability is obtained by determining the eigenvalues of the Jacobian matrix around equilibrium points. Having obtained the stable equilibrium points are then given in the form of charts and portraits simulation phase to determine the behavior of the system in the future.
\end{abstract}

Keywords: Mathematical Model, The interaction of two Populations Model, Stability

\section{Introduction}

Mathematics has many benefits in the areas of science and everyday life, especially in the field of Biology. According Widowati and Sutimin (2007:1). The mathematical model is a mathematical field that seeks to represent and describe physical systems or a problem in the real world in a mathematical statement, in order to obtain an understanding of these real world problems to be more precise. Representation of this process is known as a mathematical model. Construction analysis and use of mathematical models is seen as one of the most important applications of mathematics. Have been many studies that discuss the interaction of two species or two populations such as predator-prey or predator - prey.

Unlike the predator prey models that describe the interaction where a predator as prey and prey as prey, predators will kill its prey directly in the process of natural selection. While in this study established the relationship between host and parasitoid where the host does not kill quickly, but slowly with the way of life of a ride or take in nutrients from the host they occupy. This research is exciting to do because there are still few studies that discuss mathematical models of host and parasitoid interactions in particular discuss the stability of the system.

In this study, given how differential equations can be used to construct a mathematical model was particularly focused on the stability of differential equations mathematical model of the interaction of the two populations that host and parasitoid. Stability discussed in this study are stable equilibrium points are obtained from the characteristic equation systems of differential equations host and parasitoid interactions. Having obtained the stable equilibrium points are then given a simulation to determine the type of stable equilibrium points using matlab program.

Type the stability of the equilibrium point is determined on the eigenvalues of the Jacobian matrix. Some types of stability based on the roots of the characteristic equation is as follows. If the value of the roots of the characteristic equation real, distinct, and equally a sign of the system asymptotically stable equilibrium point. If the value of the roots of the characteristic equation complex conjugate purely imaginary but not the asymptotically stable equilibrium point. If the value of the roots of the characteristic equation real, distinct, and not as a sign of the equilibrium point of the system is unstable. If the value of the roots of the characteristic equation purely imaginary ekuilibrim point system is stable but not asymptotically stable.

Based on the interaction of host and parasitoid, a mathematical model in the form of differential equations and systems of differential equations is a suitable way to describe the interaction between host and parasitoid. Parasitoid is an organism that spends most of his life history to rely on a single host organism which eventually kills (and often took food) in the process. Parasitoids laid eggs in the body of the target animal after hatching larvae then sucking the bodily fluids of the target animal to death When the host-parasitoid find a place to live, it must be appropriate host physiology 
and nutrition for the successful development of the parasitoid.

The goal in conducting this study was to examine how the stability of differential equations of mathematical models the interaction of two populations (host and parasitoids) and examines how the stability of mathematical models simulating the interaction of the two populations of differential equations (host and parasitoid) with matlab program.

This research is the development of a mathematical model, especially the model of differential equations formed from the interaction between host and parasitoid. Applications using matlab help to find graph and phase portraits of systems of differential equations stability so they can know the behavior of the system in the future. As study materials for researchers who like or are interested in the development of mathematical models especially differential equations.

\section{The interaction of two populations models (Predator- Prey)}

The basic model Predator-Prey or predator prey interaction of the two populations is given by the equation Lotka-Volterra (1926)

$$
\begin{aligned}
& \frac{d h}{d t}=m h-a^{*} h p \\
& \frac{d p}{d t}=-q p+r a^{*} h p
\end{aligned}
$$

In this equation $h$ is the prey populations and $p$ is a predator population, with $t$ is time. The equation $\frac{d h}{d}$ states the prey population changes over time and the equation $\frac{d}{d}$ stated predator population changes over time. So the equation:

$$
N_{b}=a^{*} h T_{s}
$$

With $N_{b}$ captured prey populations of prey each unit time.

$$
f(h)=\frac{a^{*} h}{1+b h}
$$

With $b$ states the time to deal with a host, $\mathrm{f}(\mathrm{h})$ stating the number of hosts attacked parasitoid per unit time or the number of hosts diparasit, A stated figures hosted by parasitoid attack . Equation (2) is Holling type II functional response to the host parasitoid interactions.

\section{Dynamic systems}

In general, the dynamic system is defined as a real problem that is modeled mathematically using differential equations in the equation which contains parameters that are interconnected, as well as changes in the parameters of the equation will lead to changes in the stability of the equilibrium point .

The equilibrium point is one of the key concepts in a dynamic system. More general system can be expressed in the following form.

$$
\begin{gathered}
\dot{x}_{1}=f_{1}\left(x_{1}, x_{2}, \ldots, x_{n}, t\right) \\
\dot{x_{2}}=f_{2}\left(x_{1}, x_{2}, \ldots, x_{n}, t\right) \\
\vdots \\
\dot{x_{n}}=f_{n}\left(x_{1}, x_{2}, \ldots, x_{n}, t\right)
\end{gathered}
$$

With $f_{i}=\left(x_{1}, x_{2}, \ldots, x_{n}, t\right), i=1,2, \ldots, n n$ is a general function of $x_{i}, i=1,2, \ldots, n$ and time is $t$.

The system can be further simplified into a system that is not dependent on the function of time (autonomous system) as follows

$$
\begin{gathered}
\dot{x}_{1}=f_{1}\left(x_{1}, x_{2}, \ldots, x_{n}\right) \\
\dot{x_{2}}=f_{2}\left(x_{1}, x_{2}, \ldots, x_{n}\right) \\
\vdots \\
\dot{x_{n}}=f_{n}\left(x_{1}, x_{2}, \ldots, x_{n}\right)
\end{gathered}
$$

With $f_{i}=\left(x_{1}, x_{2}, \ldots, x_{n}, t\right), i=1,2, \ldots, n \mathrm{n}$ is a function that does not depend explicitly on the time $t$.

\section{The definition of equilibrium point (Perko, 1991)}

Point $\overline{x_{1}}=\left(\overline{x_{1}}, \overline{x_{2}}, \ldots, \overline{x_{n}}\right) \in R^{n}$ called the equilibrium point of (3) if $f_{l}(\bar{x})=0, i=1,2, \ldots, n$

\section{Stability of Equilibrium Point}

Definition of Local Stability the equilibrium point $x \in$ $R^{n}$ on the system (3) said:

1) Local Stable if for every $\mathrm{a}>0$ there $\ddot{a}>0$ such that for every solution $x(t)$ that satisfies $\left\|x\left(t_{0}\right)-\bar{x}\right\|<$ ä apply $\|x(t)-\bar{x}\|<$ å for each $t \geq t_{0}$.

2) Local asymptotically stable if the equilibrium point $x \in R^{n}$ stable and there $a_{0}>0$ such that for every solution $x(t)$ that satisfies $\mathrm{x}\left\|x\left(t_{0}\right)-\bar{x}\right\|<a_{0}$ apply $\lim _{t \rightarrow \infty} x(t)=\bar{x}$

3) Do not be stable if the equilibrium point $x \in R^{n}$ does not meet 1 .

Below is given a definition of linear and nonlinear systems granted system(3), With $E \subseteq R^{n}$ and $f: E \rightarrow$ $R^{n}$ continuous function on $\mathrm{E}$.

System (3) is said to be linear if $f_{1}, f_{2}, \ldots, f_{n}$ each linear with respect $x_{1}, x_{2}, \ldots, x_{n}$. so the system (3) can be written in the form

$$
\begin{aligned}
& \dot{x}_{1}=a_{11} x_{1}+a_{12} x_{2}+\cdots+a_{1 n} x_{n}, \\
& \dot{x}_{2}=a_{21} x_{1}+a_{22} x_{2}+\cdots+a_{2 n} x_{n}, \\
& \dot{x}_{n}=a_{n 1} x_{1}+a_{n 2} x_{2}+\cdots+a_{n} x_{n},
\end{aligned}
$$


With $\dot{x}_{1}=\frac{d_{i}}{d}, f_{i}$ continuous on $a \leq t \leq b, a, b$, $i=1,2, \ldots, n$. Then the system (3) can be expressed in the form $\dot{x}=A$, with $x \in E, \dot{x}=\left(\dot{x}_{1}, \dot{x}_{2}, \ldots, \dot{x}_{n}\right)$ and $\mathrm{A}$ matrix size $n \times n$.

Granted system (3) with $E \subseteq R^{n}$ and $f: E \rightarrow R^{n}$ continuous function on E. System (3) is said to be nonlinear if there is i such that $f_{i}$ nonlinear. The nature of the solution around the equilibrium point of nonlinear systems (3) can be determined through linearization around the equilibrium point of the system.

\section{Definition 2.1 Jacobian matrix}

If the function belongs $f=\left(f_{1}, f_{2}, \ldots, f_{n}\right)$ on the system (3) with $f_{i} \in C(E), i=1,2,3, \ldots, n$.

$$
\text { Matriks }\left|\begin{array}{cccc}
\frac{\partial f_{1}}{\partial x_{1}} \bar{x} & \frac{\partial f_{1}}{\partial x_{2}} \bar{x} & \ldots & \frac{\partial f_{1}}{\partial x_{n}} \bar{x} \\
\frac{\partial f_{2}}{\partial x_{1}} \bar{x} & \frac{\partial f_{2}}{\partial x_{2}} \bar{x} & \ldots & \frac{\partial f_{1}}{\partial x_{n}} \bar{x} \\
\vdots & \vdots & \ddots & \vdots \\
\frac{\partial f_{n}}{\partial x_{1}} \bar{x} & \frac{\partial f_{n}}{\partial x_{2}} \bar{x} & \ldots & \frac{\partial f_{n}}{\partial x_{n}} \bar{x}
\end{array}\right|
$$

Named Jacobian matrix of $f$ in point $\bar{x}$. (Kocak, 1991). By using Jacobian matrix $J(\bar{x})$, point stability properties ekuilibrim $\bar{x}$ it can be seen as long as the point is hyperbolic

\section{Eigenvalues and Eigenvectors}

Formally the definition of eigenvalues and eigenvectors is as follows. Definitions (Anton and Rorres, 2014)

Let A nxn matrix and $x \in R^{n}, x \neq 0$. Vector $\mathrm{x}$ is called an eigenvector / characteristic vector of $\mathrm{A}$ if

$$
A x=\ddot{\mathrm{e}} x
$$

for a $\ddot{\text { e }} \in R$. Numbers ë that satisfies the above equation is called the eigenvalues / value characteristics. Vector $\mathrm{x}$ is called an eigenvector corresponding to ë.

\section{Stability Analysis of Fixed Point (Boyce \& Diprima, 2001)}

Consider the linear system $\left(\begin{array}{l}\frac{d}{d} \\ \frac{d}{d}\end{array}\right)=\left(\begin{array}{ll}a & b \\ c & d\end{array}\right)\left(\begin{array}{l}x \\ y\end{array}\right)$ with a, $\mathrm{b}, \mathrm{c}$ and $\mathrm{d}$ constants (5).

Linear systems (5) commonly called "data model species" in population dynamics. If the matrix $\mathrm{A}=$ $\left(\begin{array}{ll}a & b \\ c & d\end{array}\right)$ and such $\lambda$ are the eigenvalues of matrix A, then:

$$
\mathrm{A}\left(\begin{array}{l}
x \\
y
\end{array}\right)=\mathrm{A}
$$

$$
\left(\begin{array}{l}
x \\
y
\end{array}\right) \leftrightarrow\left(\begin{array}{cc}
a-\ddot{\mathrm{e}} & b \\
c & d-\ddot{\mathrm{e}}
\end{array}\right)\left(\begin{array}{l}
x \\
y
\end{array}\right)=\left(\begin{array}{l}
0 \\
0
\end{array}\right)
$$

Phase portrait and linear systems (Boyce \& DiPrima, 2001)

Belongs to the following linear system

$$
\dot{x}=A
$$

Suppose $\operatorname{det}(A) \neq 0$, so $X$ are the eigenvalues of matrix $\mathrm{A}$, ie ë is the root of the characteristic equation

$$
\text { ëI }-A=0
$$

Images phase and the system (6) is almost entirely dependent on its eigenvaluesFigure. 2 shows the absorbance graphic of plot toward thin film wavelength of $\mathrm{TiO} 2$ that has been soaked in dye with variation of temperature extract.

1. If the eigenvalues different real negative is called a node, all trajectories toward zero, which means no points of zero is stable.

2. If the eigenvalues of positive reals different then called nodal source, all trajectories out of the points become unstable

3. If the eigenvalues of different real opposite in sign, with this so-called saddle point, all trajectories will be away to infinity along the eigenvectors, this resulted critical point will always be unstable.

4. If the eigenvalues of different real opposite in sign, with this so-called saddle point, all trajectories will be away to infinity along the eigenvectors, this resulted critical point will always be unstable.

5. If the eigenvalues equal, with two linearly independent eigenvectors, you will get what is called a star point or propernode, when $\lambda<0$ then the point of criticism would be stable and unstable for $\lambda>0$.

6. If the eigenvalues equal to the eigenvectors will be obtained by so-called improper node, when $\lambda<0$ then the point of criticism would be stable and directions trajectory will go to zero. Whereas for $\lambda>$ 0 directions trajectory going out leaving the zero point and the point of criticism would be unstable. If the eigenvalues are complex numbers $\ddot{e}_{ \pm}=a \pm i l$ with $a<0$, it will produce a stable behavior called Spiral,

7. If the eigenvalues are complex numbers $\ddot{e}_{ \pm}=a \pm i l$ dengan $a>0$, it will generate a behavior called unstable spiral, all trajectories going out leaving the zero point and the point of criticism would be stable. 


\section{Method}

This research is a study of Library Studies, which is used in literature studies of relevant literature sources used to gather the necessary information in the study. A literature study by collecting literature sources which may include books, texts, papers and so on. After the collected literature sources ollowed by a review of the literature sources. At the end of the library resources as a basis for analyzing the problem.

Mathematical modeling the interaction of two populations of host and parasitoid in this research is needed to determine how the shape of the equation, and then to look for form completion hosted model and the parasitoid.

Steps to resolve the problem that is used to analyze the shape and model of the study are as follows:

a. Lowering the mathematical model the interaction of two populations of host and parasitoid with Holling response function of type II.

b. Analysis of the models begins with finding the equilibrium point of the model systems of differential equations.

c. Determining the characteristic equation and the eigenvalues of the Jacobian matrix system which is calculated at each point of equilibrium.

d. Then check the stability of the equilibrium point.

e. Creating a numerical simulation of the results of the analysis of mathematical models the interaction of two populations of host and parasitoid with Holling response function of type II.

\section{Research Result}

In the discussion this time will be discussed on a mathematical model of interaction between host and parasitoid host. In this model there are two species that interact namely host and parasitoid. The population of the host is assumed to have enough food. Prior to their predators, the intrinsic growth rate (birth rate minus death rate) host proportional to its population. In this case the host becomes the main food for parasitoid so before their host, the parasitoid population growth will decline due to the mortality rate of the parasitoid.

Parasitoid attack based on the functional response of the host. In contrast with these interactions, the parasitoid population will be converted to its growth. The ratio of parasitoid immature to adult parasitoids is constant and the population has the maximum growth rate.

The parameters given to model the interaction between the host and parasitoid is $h(t)$ which states the host population at time $\mathrm{t}, p(t)$ states parasitoid population at the time, $f(h)$ stated functional response, $a$ stating the effect of oviposition on adult parasitoid population, $r$ states the intrinsic growth rate of the host ,$d$ declared the death rate of the parasitoid. All of these parameters is positive. Functional response $f(h)$ assumed to be a function $f: R^{+} \rightarrow R^{+}, \quad, f(h)$ function with a continuous rise $f(0)=0$, and $f(h)$ diferensiabel kontinu.

Based on the assumptions above were obtained host and parasitoid interaction model as follows:

$$
\begin{gathered}
\frac{d h}{d t}=r h-f(h) p \\
\frac{d p}{d t}=(a f(h)-d) p
\end{gathered}
$$

With $f(h)=\left(\frac{a^{*} h}{1+b h}\right)$ a Holling type II functional response to the interaction of host and parasitoid. Here is a system of differential equations after the interaction of the two populations substituted into the equation (8), namely:

$$
\begin{aligned}
& \frac{d h}{d}=r h-\left(\frac{a^{*} h}{1+b h}\right) p \\
& \frac{d}{d}=\left(a \frac{a^{*} h}{1+b h}-d\right) p
\end{aligned}
$$

\section{From the point Equilibrium Model}

In this section we will look for a solution to the $R_{+}^{2}$, because the population must not be negative and the solutions are beyond $R_{+}^{2}$ cannot be interpreted biologically. Equilibrium point equation (9) is obtained if:

$$
\begin{aligned}
& \frac{d h}{d t}=0 \\
& \frac{d p}{d t}=0 .
\end{aligned}
$$

From equation (10) and (11) was obtained

$$
\begin{gathered}
h\left(r-\frac{a^{*} p}{1+b h}\right)=0 \\
\left(a \frac{a^{*} h}{1+b h}-d\right) p=0 .
\end{gathered}
$$

From equation (12) and (13) was obtained 3 obtained equilibrium point

$$
\begin{aligned}
& \left(h_{1}, p_{1}\right)=\left(0, \frac{a r}{a^{*} a-d b}\right) \\
& \left(h_{2}, p_{2}\right)=\left(\frac{d}{a^{*} a-d b}, 0\right) \\
& \left(h_{3}, p_{3}\right)=\left(\frac{d}{a^{*} a-d b}, \frac{a r}{a^{*} a-d b}\right) .
\end{aligned}
$$


On the condition that the equilibrium point $a^{*} a-$ $d>0$ or $a^{*} a>d$

Based on these results it can be concluded that: if $a^{*} a>d$ then between host and parasitoid populations can coexis. $a^{*} a>d$ means that the effects of oviposition on adult parasitoid population multiplied by the time available for the parasitoids to chase and finds a host is greater than the mortality rate of parasitoid multiplied by time to handle one host. If $a^{*} a>d$ then between host and parasitoid populations cannot coexist.

\section{Stability of Equilibrium Point}

One way to determine the stability of equilibrium points of the system (9) is to use linearization. Based Systems (9), for example $h=g_{1}(x, y)$ and $p=g_{2}(x, y)$ with,

$$
\begin{gathered}
g_{1}(x, y)=r h-\left(\frac{a^{*} h}{1+b h}\right) p \text { and } \\
g_{2}(x, y)=\left(a \frac{a^{*} h}{1+b h}-d\right) p .
\end{gathered}
$$

obtainable

$$
J(h, p)=\left(\begin{array}{l}
\frac{\partial g_{1}}{\partial h} \frac{\partial g_{1}}{\partial p} \\
\frac{\partial g_{2}}{\partial h} \frac{\partial g_{2}}{\partial p}
\end{array}\right)=\left(\begin{array}{cc}
r-\frac{a^{*} p}{(1+b h)^{2}} & -\frac{a^{*} h}{1+b h} \\
\frac{a^{*} a p}{(1+b h)^{2}} & \frac{a^{*} a h}{1+b h}-d
\end{array}\right) .
$$

\section{Local Stability Analysis}

In the following theorems will discuss the local stability of equilibrium points:

$$
\begin{gathered}
\left(h_{3}, p_{3}\right)=\left(\frac{d}{a * a-d}, \frac{a}{a * a-d}\right) \\
\left(h_{1}, p_{1}\right)=\left(0, \frac{a}{a * a-d}\right) \text { and }\left(h_{2}, p_{2}\right)=\left(\frac{d}{a * a-d}, 0\right)
\end{gathered}
$$

\section{Theorem}

If $\quad a * a>d, a * a>b(d+r), \frac{a * a}{a * a-d}>r-d$, $\frac{a * a}{a * a-d}>d$ and $\left(\frac{a * a}{a * a-d}+d-r\right)^{2}<\left(\frac{a * a}{a * a-d}-r\right)$ then the equilibrium point $\left(h_{1}, p_{1}\right)=\left(0, \frac{a}{a * a-d}\right)$ local asymptotically stable.

\section{Theorem}

If $\quad a * a>d, a * a>b(d+r) \quad$ and $\left(\frac{r(a * a-d)}{a * a}-r\right)^{2}<\frac{4 r(a * a-d)}{a^{*} a}$, and $\frac{r(a * a-d)}{a * a}>r$ then the equilibrium point $\left(h_{3}, p_{3}\right)=\left(\frac{d}{a^{*} a-d b}, \frac{a r}{a^{*} a-d b}\right)$ Local asymptotically stable.

\section{Theorem}

If $\left(\frac{d}{a}+d+r\right)>0$ and $\left(\frac{d}{a}+d+r\right)^{2}>4\left(\frac{r d}{a}+r d\right)$ then the equilibrium point $\left(h_{2}, p_{2}\right)=\left(\frac{d}{a^{*} a-d b}, 0\right)$ unstable.

\section{Conclusion}

The following will be given some conclusions related to this research.

1. From the system of differential equations interaction of two species, namely:

$\frac{d h}{d}=r h-\left(\frac{a * h}{1+b h}\right) p$ and $\frac{d}{d}=\left(a \frac{a * h}{1+b h}-d\right) p$

obtained equilibrium points

$$
\begin{aligned}
& \left(h_{1}, p_{1}\right)=\left(0, \frac{a}{a * a-d}\right), \quad\left(h_{3}, p_{3}\right)=\left(\frac{d}{a * a-d}, \frac{a}{a * a-d}\right) \\
& \text { and }\left(h_{2}, p_{2}\right)=\left(\frac{a}{a * a-d}, 0\right) .
\end{aligned}
$$

2. If $a * a>d, a * a>b(d+r), \frac{a * a}{a * a-d}>r-d$, $\frac{a * a}{a * a-d}>d$

and $\left(\frac{a * a}{a * a-d}+d-r\right)^{2}<4\left(\frac{a * a}{a * a-d}, r\right)$, then the equilibrium point $\left(h_{1}, p_{1}\right)=\left(0, \frac{a}{a * a-d}\right)$ local asymptotically stable.

3. If $\left(\frac{a * a}{a * a-d}+d-r\right)^{2}<4\left(\frac{a * a}{a * a-d}, r\right)$,

$\frac{a * a}{a * a-d}>r-d$, and $\frac{a * a}{a * a-d}>d$ then

$\ddot{\mathrm{e}}_{1,2}=\frac{-\left(\frac{a^{*} a}{a^{*} a-d}+d-r\right) \pm \sqrt{\left(\frac{a^{*} a}{a^{*} a-d}+d-r\right)^{2}-4\left(\frac{a^{*} a}{a^{*} a-d}-r\right)}}{2}$

have a negative real part. So based on the equilibrium point theorem $\left(h_{1}, p_{1}\right)=\left(0, \frac{a}{a * a-d}\right)$ local asymptotically stable.

4. By Theorem if: $a * a>d, a * a>b(d+r)$, $\frac{a * a}{a * a-d}>r-d, \frac{a * a}{a * a-d}>d$ and $\left(\frac{a * a}{a * a-d}+d-\right.$ $r)^{2}<4\left(\frac{a * a}{a * a-d}-r\right)$, then at the beginning of time very small number of host population, while close to the parasitoid population $\left(\frac{a}{a * a-d}\right)$. So for a long time, the parasitoid population will approach $\left(\frac{a}{a * a-d}\right)$ while the host population will become extinct. 
5. If $a * a>d, a * a>b(d+r)$, and $\left(\frac{r(a * a-d)}{a * a}-\right.$ $r)^{2}<\frac{4 r(a * a-d)}{a * a}$, and $\frac{r(a * a-d)}{a * a}>r$ then the equilibrium point $\left(h_{3}, p_{3}\right)=\left(\frac{d}{a * a-d}, \frac{a}{a * a-d}\right)$ local asymptotically stable. Because $\left(h_{3}, p_{3}\right)=$ $\left(\frac{d}{a * a-d}, \frac{a}{a * a-d}\right)$ have negative real part, the equilibrium point $\left(h_{3}, p_{3}\right)=\left(\frac{d}{a * a-d}, \frac{a}{a * a-d}\right)$ local asymptotically stable.

By Theorem if: $\left(\frac{r(a * a-d)}{a * a}-r\right)^{2}-\frac{4 r(a * a-d)}{a * a}, a *$ $a>d, a * a>b(d+r)$, and $\frac{r(a * a-d)}{a * a}>r$, then at the beginning of time close to the host population $\frac{d}{a * a-d}$ while close to the parasitoid population $\frac{d}{a * a-d}$. So for a long time, will host population approaching $\frac{d}{a * a-d}$ and the parasitoid population will approach $\frac{a}{a * a-d}$. If $\left(\frac{d}{a}+d+r\right)>0$ and $\left(\frac{d}{a}+d+r\right)^{2}>4\left(\frac{r}{a}+r\right)$ then the equilibrium point $\left(h_{2}, p_{2}\right)=\left(\frac{a}{a * a-d}, 0\right)$ unstable.

\section{References}

Anton, H. and Rorres, C., 2014, Elementary Linear Algebra, John Wiley \& Sons, Inc., New York.

Boyce, W.E. \& DiPrima R.C. 2001. Elementary Differential Equations and Boundary Value Problems. Seventh Edition, John Wiley \& Sons: New York.

Edelstein, L., Keshet, 2005, Mathematical Models in Biology, Siam, New York.

Haberman, R. 1977. Mathematical Models in Mechanical Vibrations, Population Dynamics, and Traffic Flow. PrenticeHall: New Jersey.

Hanh, W., 1967, Stability of Motion, Springer-Verlag, New York.

Hale, J. K. \& H. Kocak. 1991. Dynamics and bifurcations. New York: Springer Verlag.

Kuznetsov, Y.A., 1998, Elements of Applied bifurcation Theory, Springer-Verlag, New York.

Ni'mah, Khoirun, 2015, Analysis of Model S-I-P Interaction of Two Species Predator-Prey With Response Function Holling Type II, Semarang State University.

Perko, L., 2001. Differential Equations and Dynamical Systems, Springer-Verlag, New York.

Sulistyowati, E. et al., 2001, Functional Response Parasitoid Cephalonomia Stephanoderis Betr Against Coffee Fruit powder, Hypothenemus hampei Ferr, Pest Plant Science Program, University of Gadjah Mada.

Tarumingkeng, R.C., Population Dynamics (Quantitative Ecological Assessment), 1994, Pustaka Sinar Harapan and Activities of Discourse Christian University, Jakarta. 